\title{
BORELIAN DENSITIES IN NUMBER THEORY: BORELIAN DENSITIES OF ARITHMETICAL FUNCTIONS
}

\section{NOUREDDINE DAILI}

Department of Mathematics

Cité Des 300 Lots, Yahiaoui

51 Rue Harrag Senoussi

19000 Sétif

Algeria

e-mail: nourdaili_dz@yahoo.fr

\begin{abstract}
In this work, in consideration of the classical Poisson probabilistic law, we introduce Borelian density of arithmetical function and make comparative study of this density with others. These results represent a generalization of results of ([1]). Further, we provide certain criteria and also the applications of this newly proposed density.
\end{abstract}

\section{Introduction}

The probabilistic Poisson law is the law of random variables which count the number of occurrences of a rare event. Its domain of application was limited for a long time to that of rare event. But, fore some decades, its field of application considerably widened. It got used in 2010 Mathematics Subject Classification: Primary 11B05, 11R45; Secondary 11K65, 60A10, $60 \mathrm{~B} 10$.

Keywords and phrases: probabilistic law, density, arithmetical function, number theory.

Received September 30, 2016

(C) 2016 Scientific Advances Publishers 
telecommunications, the statistical quality control, study of the phenomena connected to the radioactive splitting, the biology, the medicine, the meteorology, the industry and elsewhere.

The network of Poisson probability is met under diverse forms. That is, in several contexts. It is defined for all the subsets of all the whole natural integers set $\mathbb{N}=\{0,1,2, \ldots\}$ and for all positive bounded arithmetical functions.

Throughout the paper, $f$ denotes a positive bounded arithmetical function.

In this work, we consider the family, of measures of probability on $\mathbb{N}$, defined by

$$
b_{\lambda}(f ; k)=\frac{1}{k !} \lambda^{k} e^{-\lambda} f(k), \quad k \in \mathbb{N} .
$$

Definition 1.1. The family of Borelian or Poisson probability measures on the arithmetic function $\mathrm{f}$ is defined as follows:

$$
b_{\lambda}(f ; k):=\frac{1}{k !} \lambda^{k} e^{-\lambda} f(k), \text { for } k=0,1, \ldots
$$

These can be made diffuse by letting $\lambda \nearrow+\infty$.

We calculate the measure of probability of an arithmetical function $f$. Then we diffuse this measure by passing onto the limit on $\lambda$, as $\lambda$ goes to $+\infty$, namely, $\lambda \nearrow+\infty$, we obtain what we shall call the Borelian density of $f$.

We are going to show that the binomial density implies the Borelian density for positive and bounded arithmetical functions. The converse is false generally. So, if we denote a class of positive and bounded arithmetical functions which admit binomial density by $\mathcal{B}$ and a class of positive and bounded arithmetical functions which admit a Borelian density by $\mathcal{P}$, then we obtain a strict inclusion $\mathcal{B} \subset \mathcal{P}$.

The Borelian density related to Poisson probabilistic law for a positive bounded arithmetical function is strictly defined in what follows. 


\section{Main Results}

\subsection{Borelian density of arithmetical functions}

We calculate the measure of probability of a positive and bounded arithmetical function $f$. Then we diffuse this measure by passing onto the limit on $\lambda$, as $\lambda$ goes to $+\infty$, namely, $\lambda \nearrow+\infty$. Thus, we obtain the Borelian density.

We then study the link of these densities with the binomial, asymptotic and $H^{p}, p \in \mathbb{N}^{*}$ densities introduced in ([2], [4], and [5]) for arithmetical functions. More precisely, we show that the binomial density implies the Borelian density for positive and bounded arithmetical functions. The converse is false, in general. So, if we denote a class of positive and bounded arithmetical functions which admits binomial density by $\mathcal{B}$ and a class of positive and bounded arithmetical functions which admits a Borelian density by $\mathcal{P}$, then we obtain a strict inclusion $\mathcal{B} \subset \mathcal{P}$.

Definition 2.1. Let

$$
B_{\lambda}(f):=e^{-\lambda} \sum_{k \geq 0} \frac{\lambda^{k}}{k !} f(k) .
$$

We say that $f$ has the number $\ell,(\ell \geq 0)$, as the Borelian density related to the Poisson measure probabilistic law if limit $\lim _{\lambda} B_{\lambda}(f)$ exists and equals $\ell$, as $(\lambda \nearrow+\infty)$. If it is the case, we shall denote this density by $\beta(f)$ and write

$$
\beta(f)=\lim _{(\lambda \nearrow+\infty)} B_{\lambda}(f)=\ell
$$

We call that $\beta(f)$ is the Borelian density of $f$.

Let us give theorems characterizing the Borelian density of the positive and bounded arithmetical functions in touch with the binomial and asymptotic densities. 
Theorem 2.1 (Comparison between densities). If $f$ admits a binomial density $\mathfrak{b}(f)([2])$, then it has the Borelian density $\beta(f)$ and the two limit values are equal, namely,

$$
\mathfrak{b}(f)=\beta(f)=\ell \geq 0 .
$$

Proof. Suppose $f$ has a binomial density $\ell$. Then

$$
\lim _{(n \rightarrow+\infty)} B_{n}(f ; p)=\ell
$$

We have

$$
\begin{gathered}
e^{-\lambda} \sum_{k} \frac{\lambda^{k}}{k !} f(k) \rightarrow \ell, \text { as }(\lambda \nearrow+\infty) \\
\Uparrow \\
e^{-p \lambda} \sum_{k} \frac{(p \lambda)^{k}}{k !} f(k) \rightarrow \ell, \text { as }(\lambda \nearrow+\infty) .
\end{gathered}
$$

Moreover,

$$
e^{q \lambda} \sum_{k} \frac{(p \lambda)^{k}}{k !} f(k)=\left(\sum_{j} \frac{(q \lambda)^{j}}{j !}\right)\left(\sum_{k} \frac{(p \lambda)^{k}}{k !} f(k)\right)=\sum_{n} c(n) \lambda^{n},
$$

where

$$
c(n)=\frac{q^{n}}{n !} f(0)+\frac{p q^{n-1}}{(n-1) !} f(1)+\ldots+\frac{p^{n}}{n !} f(n)
$$

or

$$
c(n)=\frac{1}{n !} B_{n}(f ; p)
$$

Therefore,

$$
e^{-p \lambda} \sum_{k} \frac{(p \lambda)^{k}}{k !} f(k)=e^{-p \lambda} e^{-q \lambda} e^{q \lambda} \sum_{k} \frac{(p \lambda)^{k}}{k !} f(k)=e^{-\lambda} \sum_{n} B_{n}(f ; p) \frac{\lambda^{n}}{n !} .
$$


But

$$
\lim _{(n \rightarrow+\infty)} B_{n}(f ; p)=\ell
$$

according the Toeplitz Lemma ([6], Chapter 3, p. 43). Hence

$$
e^{-\lambda} \sum_{n} B_{n}(f ; p) \frac{\lambda^{n}}{n !} \rightarrow \ell, \text { as }(\lambda \nearrow+\infty) .
$$

So, the arithmetical function $f$ has the Borelian density $\beta(f)=\ell$.

Corollary 2.2. Let $E \neq \emptyset$ be a subset of $\mathbb{N}$. If $E$ admits a binomial density $\mathfrak{b}(E)([2])$, then it has a Borelian density $\beta(E)$ ([1]) and the two limit values are equal, namely, $\mathfrak{b}(E)=\beta(E)=\ell$.

Proof. Results from the application of the previous Theorem 2.1 by taking $f(k)=I_{E}(k)$ and applying the Theorem 2.1 ([1]).

Remark 2.1. It is not well known, at the moment, if the binomial density is strictly weak that the Borelian density for a positive bounded arithmetical function.

Definition 2.2. We have

(a) Césaro summability means that:

$$
\sum_{k} f(k)=\ell(C ; s)
$$

(b) Hölder's summability means that:

$$
\sum_{k} f(k)=\ell(H ; s)
$$

We need following theorems connecting the asymptotic density and the $H^{p}$-density for $p \in \mathbb{N}^{*}$ for an arithmetical function $f$ : 
Theorem 2.3 ([4], [5]). Let $f: \mathbb{N} \rightarrow \mathbb{R}^{+}$be a positive and bounded arithmetical function. Then for all real number $\ell \geq 0$, the following two statements are equivalent:

$\left(\mathrm{p}_{1}\right) f$ admits $\ell$ as $H^{1}$-density and $\left(d_{1}(f)=\ell\right)$;

$\left(\mathrm{p}_{2}\right) f$ admits $\ell$ as $H^{p}$-density, for all $p \in \mathbb{N}^{*}$ and $\left(d_{p}(f)=\ell\right)$.

More generally, we have the following theorem:

Theorem 2.4. For all real number $\ell \geq 0$ and $s \in \mathbb{N}^{*}$, the following two statements are equivalent:

$\left(\mathrm{p}_{1}\right) f$ admits $\ell$ as an asymptotic density of order $s$ and $\left(d_{s}^{C}(f)=\ell\right)$;

$\left(\mathrm{p}_{2}\right) f$ admits $\ell$ as $H^{s}$-density and $\left(d_{s}^{H}(f)=\ell\right)$.

Theorem 2.5. If $f(n)=o(\sqrt{n})$ and $f$ admits a Borelian density $\beta(f)$, then $f$ admits an asymptotic density $d(f)$ of order $2\left(d_{2}(f)=d_{2}^{C}(f)=\ell\right)$.

Theorem 2.6. If $f$ admits the Borelian density $\beta(f)=\ell$, then it admits an asymptotic density $d(f)$ and the two limit values are equal, namely,

$$
\beta(f)=d(f)=\ell \geq 0
$$

Proof. From Borel summability,

$$
\sum_{k} f(k)=\ell(B)
$$

This implies that

$$
e^{-\lambda} \sum_{k \geq 0} f(k) \frac{\lambda^{k}}{k !} \rightarrow \ell, \quad \text { as }(\lambda \nearrow+\infty) .
$$

Thus, a positive and bounded arithmetical function $f: \mathbb{N} \rightarrow \mathbb{R}^{+}$has an asymptotic density $\ell$ if, and only if, 


$$
\sum_{k} f(k)=\ell(C ; 1)=d(f)
$$

where

$$
f(k)=\Delta a(k)=a(k)-a(k-1) .
$$

To see this, consider

$$
\sum_{k} f(k)=\ell(C ; 1) \Leftrightarrow \frac{\sum_{k=0}^{n} B(k)}{n+1} \rightarrow \ell \Leftrightarrow \frac{\sum_{k=0}^{n} f(k)}{n+1} \rightarrow \ell .
$$

Suppose $f$ admits the Borelian density $\ell$. Because $B(k)=a(k)$ means that

$$
e^{-\lambda} \sum_{k} B(k) \frac{\lambda^{k}}{k !} \rightarrow \ell, \text { as }(\lambda \nearrow+\infty) \text {. }
$$

Also,

$$
\sum_{k} f(k)=\ell(B)
$$

also $f(n)=0$ or +1 .

Thus, from Theorems 2.3 and 2.4, we have

$$
\sum_{k} f(k)=\ell(C ; 2)
$$

and hence

$$
\sum_{k} f(k)=\ell(H ; 2)
$$

Finally, according to ([4], Theorem 2.1, p. 63) or Theorem 2.3 above, we have

$$
\sum_{k} f(k)=\ell(C ; 1)
$$

Thus, $f$ admits an asymptotic density. 
Corollary 2.7. Let $E \neq \emptyset$ be a subset of $\mathbb{N}$. If $E$ admits the Borelian density $\beta(E)$, then it has an asymptotic density $d(E)$ and the two limit are equal, namely, $\beta(E)=d(E)=\ell$.

Proof. Results from the application of the previous Theorem 2.6 by taking $f(k)=I_{E}(k)$ and applying the Theorem 2.6 ([1]).

\subsection{Applications}

Theorem 2.8. Let $f: \mathbb{N} \rightarrow \mathbb{R}$ be an arithmetical function. If

$$
\sum_{k=0}^{n} f(k)=\ell n+O(g(n))
$$

where $g$ varies regularly with exponent $\alpha, 0 \leq \alpha \leq \frac{1}{2}$, then $f$ admits the Borelian density $\beta(f)$ and $\beta(f)=\ell$.

Proof. Results from the application of Theorem 2.12 ([2], p. 142) and Theorem 2.6 above.

Theorem 2.9. Let $f$ be a positive arithmetical function such that $\lim _{(n \rightarrow+\infty)} f(n)=\ell$. Then $f$ admits the Borelian density $\beta(f)$ and $\beta(f)=\ell$.

The converse of this theorem is not true, in general. However, we have

Theorem 2.10. Let $f: \mathbb{N}^{*} \rightarrow \mathbb{R}$ be an arithmetical function such that

$$
n(f(n)-f(n+1))<M,
$$

where $M>0$. Iffadmits the Borelian density $\beta(f)=\ell$, then $\lim _{(n \rightarrow+\infty)} f(n)=\ell$.

Proof. Follows from Theorem 2.3 ([5], p. 340) and Theorem 2.6 above.

We have also, 
Proposition 2.11. Let $f: \mathbb{N}^{*} \rightarrow \mathbb{R}$ be an arithmetical function. If $f$ has the Borelian density $\beta(f)=\ell$ and if the product $n(f(n)-f(n+1))$ grows, then $\lim _{(n \rightarrow+\infty)} f(n)=\ell$.

Remark 2.2. The problem of criteria remains opened for arithmetic functions and that goes to a heuristic sense.

\section{Acknowledgements}

Author would like to thank anonymous referees for their very careful reading of the manuscript.

\section{References}

[1] N. Daili, Borelian densities in number theory: Borelian densities of subsets of $\mathbb{N}$, JP Jour. Algebra, Number Theory \& Appl. (JPANTA) 38(3) (2016), 261-270.

http://dx.doi.org/10.17654/NT038030261

[2] N. Daili, Binomial densities in number theory, Jour. Algebra and Discrete Structures (JADS) 6(3) (2006), 135-143.

[3] N. Daili, Binomial densities related to Abelian-Tauberian theorems, JP Jour. Algebra, Number Theory \& Appl. (JPANTA) 15(2) (2009), 163-170.

[4] N. Daili, $H^{p}, p \in \mathbb{N}^{*}, H^{\infty}$-densities in number theory, Part II: $H^{p}, p \in \mathbb{N}^{*}$, $H^{\infty}$-densities of arithmetic functions, JP Jour. Algebra, Number Theory \& Appl. (JPANTA) 9(1) (2007), 81-93.

[5] N. Daili, Asymptotic densities in number theory, Part II: Asymptotic densities of arithmetic functions, JP Jour. Algebra, Number Theory \& Appl. (JPANTA) 6(2) (2006), 335-360.

[6] G. H. Hardy, Divergent Series, Oxford University Press, Oxford, 1963. 